\title{
Exactly solvable models and spontaneous symmetry breaking *
}

\begin{abstract}
We study a few two-dimensional models with massless and massive fermions in the hamiltonian framework and in both conventional and light-front forms of field theory. The new ingredient is a modification of the canonical procedure by taking into account solutions of the operator field equations. After summarizing the main results for the derivative-coupling and the Thirring models, we briefly compare conventional and light-front versions of the Federbush model including the massive current bosonization and a Bogoliubov transformation to diagonalize the Hamiltonian. Then we sketch an extension of our hamiltonian approach to the two-dimensional Nambu - Jona-Lasinio model and the Thirring-Wess model. Finally, we discuss the Schwinger model in a covariant gauge. In particular, we point out that the solution due to Lowenstein and Swieca implies the physical vacuum in terms of a coherent state of massive scalar field and suggest a new formulation of the model's vacuum degeneracy.
\end{abstract}

Keywords solvable relativistic models · operator solutions · vacuum structure · Bogoliubov transformation · spontaneous symmetry breaking

\section{Introduction}

Exactly solvable models may seem to be almost a closed chapter in the development of quantum field theory. Our original aim for looking at this class of simple relativistic theories was to learn about their properties in the light-front (LF) formulation and compare that picture with the standard one (by the latter we mean conventional operator formalism in terms of usual space-like (SL) field variables). Surprisingly enough, a closer look at these models revealed certain inconsistencies and contradictions in the known SL solutions. For example, the Fock vacuum was taken as the true ground state for calculations of the correlation functions in the Thirring model [1] although a derivation of the model's Hamiltonian shows that it is non-diagonal when expressed in terms of corresponding creation and annihilation operators. Hence the Fock vacuum cannot be its (lowest-energy) eigenstate. Another example is the (massless) Schwinger model, often invoked as a prototype for more complicated gauge theories. It turns out that the widely-accepted covariant-gauge solution 2 involves some unphysical degrees of freedom as a consequence of residual gauge freedom. A rigorous analysis 3 in the axiomatic spirit showing some spurious features of the solution [2] remained almost unnoticed and a less formal, e.g. a hamiltonian study correcting the physical picture seems to be lacking in literature.

Defining property of the soluble models is that one can write down operator solutions of the field equations. Hence, one should be able to extract their physical content completely. A novel feature that

\footnotetext{
* Presented by the author at LIGHTCONE 2011, 23-27 May, 2011, Dallas
} 
has been overlooked so far is a necessity to formulate these models in terms of true field degrees of freedom - the free fields. As we show below, the operator solutions are always composed from free fields. One should take this information into account by re-expressing the Lagrangiam and consequently the Hamiltonian in terms of these variables. One can summarize the above statement by a slogan: "work with the right Hamiltonian!" Recently, we applied this strategy to the massive derivative-coupling model (DCM), the Thirring and the Federbush models. The brief summary of the achieved results is given in the following section. Then we extend our approach to the case of the chiral Gross-Neveu model, the Thirring-Wess and the Schwinger model. Not all the details are worked out at the present stage - we formulate the main ideas and indicate the strategy to be followed. The full treatment of the models will be given separately [4. We conclude the present paper with a brief description of the symmetry-breaking pattern fully based on the light-front dynamics.

\section{Hamiltonian study of three models - DCM, Thirring and Federbush}

The model with derivative coupling [5] turns out to be almost a trivial one. Its Lagrangian

$$
\mathcal{L}=\frac{i}{2} \bar{\Psi} \gamma^{\mu} \stackrel{\leftrightarrow}{\partial_{\mu}} \Psi-m \bar{\Psi} \Psi+\frac{1}{2} \partial_{\mu} \phi \partial^{\mu} \phi-\frac{1}{2} \mu^{2} \phi^{2}-g \partial_{\mu} \phi J^{\mu}, \quad J^{\mu}=\bar{\Psi} \gamma^{\mu} \Psi
$$

leads to the Dirac equation which is explicitly solved in terms of a free scalar and fermion field:

$$
\Psi(x)=: e^{i g \phi(x)}: \psi(x), \quad i \gamma^{\mu} \partial_{\mu} \psi(x)=m \psi(x) .
$$

The massive scalar field $\phi(x)$ obeys the free Klein-Gordon equation as a consequence of the current conservation. The conventional canonical treatment yields a surprising result: the obtained LF Hamiltonian is a free one while the SL Hamiltonian contains an interacting piece, which is non-diagonal in terms of Fock operators and hence its true ground state (which can be obtained by a Bogoliubov transformation) differs from the Fock vacuum. So the physical pictures in two quantization schemes contradict each other. The explanation is simple. One observes that the solution (2) means that there is no independent interacting field - it is composed from the free fields. We have to insert the solution to the Lagrangian first (analogously to inserting a constraint into a Lagrangian), then calculate conjugate momenta and derive the Hamiltonian. In this way, a free Lagrangian and Hamiltonian are found also in the SL case. The new procedure does not alter the LF result. The correlation functions in the two schemes coincide as well. They are built from free scalar and fermion two-point functions.

The Thirring model [6] with its Lagrangian describing a self-interacting massless Fermi field

$$
\mathcal{L}=\frac{i}{2} \bar{\Psi} \gamma^{\mu} \stackrel{\leftrightarrow}{\partial_{\mu}} \Psi-\frac{1}{2} g J_{\mu} J^{\mu}, \quad J^{\mu}=\bar{\Psi} \gamma^{\mu} \Psi
$$

is a more complicated theory. The simplest solution is similar to Eq.(2) but the elementary scalar field $\phi$ is replaced by the composite field $j(x)$ defined via $J_{\mu}=j_{\mu}=\frac{1}{\sqrt{\pi}} \partial_{\mu} j$. The corresponding Hamiltonian $H$ is non-diagonal in composite boson operators $c, c^{\dagger}$ built from fermion bilinears according to

$$
\begin{aligned}
& j^{\mu}(x)=-\frac{i}{\sqrt{2} \pi} \int \frac{d k^{1}}{\sqrt{2 k^{0}}} k^{\mu}\left\{c\left(k^{1}\right) e^{-i \hat{k} \cdot x}-c^{\dagger}\left(k^{1}\right) e^{i \hat{k} \cdot x}\right\} \\
& c\left(k^{1}\right)=\frac{i}{\sqrt{k^{0}}} \int d p^{1}\left\{\theta\left(p^{1} k^{1}\right)\left[b^{\dagger}\left(p^{1}\right) b\left(p^{1}+k^{1}\right)-(b \rightarrow d)\right]+\epsilon\left(p^{1}\right) \theta\left(p^{1}\left(p^{1}-k^{1}\right)\right) d\left(k^{1}-p^{1}\right) b\left(p^{1}\right)\right\} .
\end{aligned}
$$

A diagonalization by a Bogoliubov transformation $U H U^{-1}$ implemented by a unitary operator $U[\gamma(g)]$, where $\gamma$ is a suitably chosen function, generates the true ground state as

$$
|\Omega\rangle=N \exp \left\{-\kappa \int_{-\infty}^{+\infty} \mathrm{d} p^{1} c^{\dagger}\left(p^{1}\right) c^{\dagger}\left(-p^{1}\right)\right\}|0\rangle .
$$

Here $N$ is a normalization factor and $\kappa$ is a $g$-dependent function. $|\Omega\rangle$ corresponds to a coherent state of pairs of composite bosons with zero values of the total momentum, charge and axial charge. Thus no chiral symmetry breaking occurs in the model at least for $g \leq \pi$ where the diagonalization is valid. 
The Federbush model [7] is the only known massive solvable model. The solvability comes from a specific current-current coupling between two species of massive fermions described by the Lagrangian

$$
\mathcal{L}=\frac{i}{2} \bar{\Psi} \gamma^{\mu} \overleftrightarrow{\partial_{\mu}} \Psi-m \bar{\Psi} \Psi+\frac{i}{2} \bar{\Phi} \gamma^{\mu} \overleftrightarrow{\partial_{\mu}} \Phi-\mu \bar{\Phi} \Phi-g \epsilon_{\mu \nu} J^{\mu} H^{\nu}
$$

Here the currents are $J^{\mu}=\bar{\Psi} \gamma^{\mu} \Psi, H^{\mu}=\bar{\Phi} \gamma^{\mu} \Phi$. The coupled field equations

$$
i \gamma^{\mu} \partial_{\mu} \Psi(x)=m \Psi(x)+g \epsilon_{\mu \nu} \gamma^{\mu} H^{\nu}(x) \Psi(x), \quad i \gamma^{\mu} \partial_{\mu} \Phi(x)=\mu \Phi(x)-g \epsilon_{\mu \nu} \gamma^{\mu} J^{\nu}(x) \Phi(x)
$$

have the solution of the form (2) with two "integrated currents" $j(x)$ and $h(x)$. One again finds that the structure of the SL and LF Hamiltonians coincides only when the operator solution is implemented in the Lagrangians. However, the SL Hamiltonian is not diagonal and a Bogoliubov transformation is needed to find the physical ground state. This requires a generalization of Klaiber's massless bosonization yielding a complicated substitute for $c\left(k^{1}\right)$ of Eq.(5). In a sharp contrast, the LF massive bosonization is as simple as the SL massless one. The model is very suitable for a non-perturbative comparison of the two forms of the relativistic dynamics. This is because 2-D massless fields cannot be treated directly in the LF formalism (only as the massless limits of massive theories - this is obvious already from the LF massive two-point functions). Exponentials of the massive composite fields are more singular than the massless once. They have to be defined using the "triple-dot ordering" 8, 9 which generalizes the normal ordering (subtractions of the VEVs order by order). We avoid this by bosonization of the massive current. The price we pay is complicated commutators at unequal times that are needed for computation of correlation functions. In any case, the correlators in the SL and LF version of the theory should coincide in form. A remarkable albeit for the moment only a conjectured scenario is that this will indeed happen with complicated operator structures plus non-trivial vacuum structure in the SL case and with much simpler operator part plus the Fock vacuum in the LF case.

\section{Chiral Gross-Neveu model}

The Lagrangian and field equations of the chiral Gross-Neveu model [10] are

$$
\mathcal{L}=\frac{i}{2} \bar{\Psi} \gamma^{\mu} \stackrel{\leftrightarrow}{\partial_{\mu}} \Psi-\frac{g}{2}\left[(\bar{\Psi} \Psi)^{2}+\left(\bar{\Psi} i \gamma^{5} \Psi\right)^{2}\right], \quad i \gamma^{\mu} \partial_{\mu} \Psi=g\left[(\bar{\Psi} \Psi) \Psi-\left(\bar{\Psi} \gamma^{5} \Psi\right) \gamma^{5} \Psi\right]
$$

This theory is a 2-D version of the Nambu-Jona-Lasinio model. When rewritten in the component form, one realizes that the above equations up to the sign coincide with those in the Thirring model:

$$
\begin{gathered}
i\left(\partial_{0}-\partial_{1}\right) \Psi_{1}=2 g \Psi_{2}^{\dagger} \Psi_{1} \Psi_{2}=-2 g \Psi_{2}^{\dagger} \Psi_{2} \Psi_{1}=-g\left(j^{0}+j^{1}\right), \\
i\left(\partial_{0}+\partial_{1}\right) \Psi_{2}=2 g \Psi_{1}^{\dagger} \Psi_{2} \Psi_{1}=-2 g \Psi_{1}^{\dagger} \Psi_{1} \Psi_{2}=-g\left(j^{0}-j^{1}\right) .
\end{gathered}
$$

Inserting the solution known from the Thirring model into the Lagrangian, we find the Hamiltonian

$$
H=\int_{-\infty}^{+\infty} \mathrm{d} x^{1}\left\{-i \psi^{\dagger} \alpha^{1} \partial_{1} \psi+\frac{1}{2} g\left(j^{0} j^{0}-j^{1} j^{1}\right)+\frac{g}{2}\left[(\bar{\psi} \psi)^{2}-\left(\bar{\psi} \gamma^{5} \psi\right)^{2}\right] .\right.
$$

We can bosonize the scalar densities $\Sigma(x)=\bar{\psi} \psi=\psi_{1}^{\dagger} \psi_{2}+\psi_{2}^{\dagger} \psi_{1}, \Sigma_{5}(x)=i \bar{\psi} \gamma^{5} \psi=i\left(\psi_{1}^{\dagger} \psi_{2}-\psi_{2}^{\dagger} \psi_{1}\right)$ :

$$
\Sigma(x)=\int_{-\infty}^{+\infty} \frac{d k^{1}}{2 \pi}\left[A\left(k^{1}, t\right) e^{i k^{1} x^{1}}+A^{\dagger}\left(k^{1}, t\right) e^{-i k^{1} x^{1}}\right], \Sigma_{5}(x)=\int_{-\infty}^{+\infty} \frac{d k^{1}}{2 \pi}\left[B\left(k^{1}, t\right) e^{i k^{1} x^{1}}+H . c .\right]
$$

With the Fock expansions for the Fermi field and after the Fourier transform, we obtain

$$
\begin{aligned}
A\left(k^{1}, t\right)= & \int_{-\infty}^{+\infty} \mathrm{d} p^{1}\left\{\frac{1}{2}\left[b^{\dagger}\left(-p^{1}\right) b\left(k^{1}-p^{1}\right)+d^{\dagger}\left(-p^{1}\right) d\left(k^{1}-p^{1}\right)\right] \theta\left(p^{1}\left(k^{1}-p^{1}\right)\right) e^{i 2 p^{1} t}\right. \\
& \left.-\theta\left(p^{1} k^{1}\right) \epsilon\left(p^{1}\right)\left[d\left(-p^{1}\right) b\left(p^{1}+k^{1}\right)+b\left(-p^{1}\right) d\left(p^{1}+k^{1}\right)\right] e^{-i 2 p^{1} t}\right\} e^{-i k^{1} t}
\end{aligned}
$$


and similarly for $B\left(k^{1}, t\right)$. One then has to diagonalize the Hamiltonian $H=H_{0}+H_{1}+H_{2}$ where

$$
\begin{aligned}
& H_{1}=-\frac{g}{\pi} \int_{-\infty}^{+\infty} d k^{1}\left|k^{1}\right|\left[c^{\dagger}\left(k^{1}\right) c^{\dagger}\left(-k^{1}\right)+c\left(k^{1}\right) c\left(-k^{1}\right)\right], \\
& H_{2}=-\frac{g}{4 \pi} \int_{-\infty}^{+\infty} d k^{1}\left|k^{1}\right|\left\{\left[2 A^{\dagger}\left(k^{1}\right) A\left(k^{1}\right)+A^{\dagger}\left(k^{1}\right) A^{\dagger}\left(-k^{1}\right)+A\left(k^{1}\right) A\left(-k^{1}\right)\right]+[A \rightarrow B]\right\} .
\end{aligned}
$$

Obviously $|0\rangle$ is not an eigenstate of $H$. The true vacuum has to be found by a Bogoliubov transformation. It will be different than the Thirring-model vacuum and probably non-invariant under $Q_{5}$. This remains to be verified. We also leave for future work generalization of the model to $N_{f}$ flavours.

\section{Thirring-Wess model}

This model 11 is simpler than the Schwinger model because the nonzero bare mass of the vector field removes gauge invariance with all its subtleties. The corresponding Lagrangian

$$
\mathcal{L}=\frac{i}{2} \bar{\Psi} \gamma^{\mu} \stackrel{\leftrightarrow}{\partial_{\mu}} \Psi-\frac{1}{4} G_{\mu \nu} G^{\mu \nu}+\mu_{0}^{2} B_{\mu} B^{\mu}-e J_{\mu} B^{\mu}, \quad G_{\mu \nu}=\partial_{\mu} B_{\nu}-\partial_{\nu} B_{\mu}
$$

leads to the field equations - the Dirac and Proca equations:

$$
i \gamma^{\mu} \partial_{\mu} \Psi(x)=e \gamma^{\mu} B_{\mu}(x) \Psi(x), \quad \partial_{\mu} G^{\mu \nu}(x)+\mu_{0}^{2} B^{\mu}(x)=e J^{\mu}(x) .
$$

The rhs of the second equation reduces to $\left(\partial_{\nu} \partial^{\nu}+\mu_{0}^{2}\right) B^{\nu}$ since $\partial_{\nu} B^{\nu}=0$ due to the current conservation. The latter condition also permits us to write down the solution of the corresponding Dirac equation as

$$
\Psi(x)=\exp \left\{-\frac{i e}{2} \gamma^{5} \int_{-\infty}^{+\infty} \mathrm{d} y^{1} \epsilon\left(x^{1}-y^{1}\right) B^{0}\left(y^{1}, t\right)\right\} \psi(x), \quad \gamma^{\mu} \partial_{\mu} \psi(x)=0 .
$$

Product of two fermion operators has to be regularized by a point-splitting. The integral in the exponent contributes naturally to find ( $j^{\mu}$ and $j_{5}^{\mu}$ are the free currents)

$$
J^{\mu}(x)=j^{\mu}(x)-\frac{e}{\pi} B^{\mu}(x), \quad J_{5}^{\mu}(x)=j_{5}^{\mu}(x)-\frac{e}{\pi} \epsilon^{\mu \nu} B_{\nu}(x) .
$$

Inserting the above $J^{\mu}(x)$ to the Proca equation, one finds that the the bare mass is replaced by $\mu^{2}=\mu_{0}^{2}+e^{2} / \pi$ and that this equation can be easily inverted since only the free fields are involved. Thus, there is no dynamically independent vector field. Following our method, we insert the solutions for $B^{\mu}$ and $\Psi$ into Lagrangian and then derive the Hamiltonian. The question if the latter will be diagonal or will have to be diagonalized, together with other properties of the model, is under study.

\section{The Schwinger model in the Landau gauge}

The masslessness of the vector field makes the Schwinger model more subtle than was the previous one. The key question is to correctly handle the gauge variables since in the covariant gauge $\partial_{\mu} A^{\mu}=0$ not all gauge freedom has been removed. We implement the gauge condition in the Lagrangian as [12]:

$$
\mathcal{L}=\frac{i}{2} \bar{\Psi} \gamma^{\mu} \overleftrightarrow{\partial_{\mu}} \Psi-\frac{1}{4} F_{\mu \nu} F^{\mu \nu}-e J_{\mu}(x) A^{\mu}(x)-G(x) \partial_{\mu} A^{\mu}(x)+\frac{1}{2}(1-\gamma) G^{2}(x), \quad F_{\mu \nu}=\partial_{\mu} A_{\nu}-\partial_{\nu} A_{\mu}
$$

The gauge-fixing terms furnish the component $A^{0}$ with the conjugate momentum, $\Pi_{A^{0}}(x)=-G(x)$. Moreover, they guarantee restriction to an arbitrary covariant gauge in which neither the condition $\partial_{\mu} A^{\mu}(x)=0$ nor the Maxwell equations $\partial_{\mu} F^{\mu \nu}(x)=e J^{\nu}(x)$ hold as operator relations. The gauge fixing field obeys $\partial_{\mu} \partial^{\mu} G(x)=0$ so that positive and negative-frequency parts $G^{( \pm)}(x)$ are well defined. 
Our strategy is to proceed in the spirit of K. Haller's generalization [12] of the Gupta-Bleuler quantization, in which the unphysical components of the gauge field are represented as ghost degrees of freedom of zero norm, carrying vanishing momentum and energy. To ensure that we are dealing with the original 2-dimensional QED, we have to restrict the theory to the physical subspace $G^{(+)}|p h y s\rangle=0$.

We will choose $\gamma=1$ in the above Lagrangian. Then the gauge condition is an operator relation while the (modified) Maxwell and Dirac equations read

$$
\partial_{\mu} F^{\mu \nu}(x)=e J^{\nu}(x)-\partial^{\nu} G(x), \quad i \gamma^{\mu} \partial_{\mu} \Psi(x)=e \gamma_{\mu} A^{\mu}(x) \Psi(x) .
$$

The solution of the latter is completely analogous to the Thirring-Wess model case, Eq.(17). Again, the vector and axial-vector currents have to be calculated via point-splitting with an important difference: the exponential of the line integral of the gauge field must be inserted in the current definition to compensate for the violation of gauge invariance due to the point splitting. After inserting the calculated interacting current into the Maxwell equations, we have to express the Lagrangian and Hamiltonian in terms of the free fields as before. The physical picture will become transparent if we make a unitary transformation to the Coulomb-gauge representation [12. Before performing this step, let us indicate the main ingredients of the original covariant-gauge solution [2,13] and point out a problem with it.

The starting point was the Ansatz for the gauge field and the currents, $\left(\tilde{\partial}_{\mu}=\epsilon_{\mu \nu} \partial^{\nu}\right)$

$$
A_{\mu}=-\frac{\sqrt{\pi}}{e}\left(\tilde{\partial}_{\mu} \Sigma+\partial_{\mu} \tilde{\eta}\right), \quad J^{\mu}=\bar{\Psi} \gamma^{\mu} \Psi=-\frac{1}{\sqrt{\pi}} \tilde{\partial}^{\mu} \Phi, \quad J_{5}^{\mu}=\bar{\Psi} \gamma^{\mu} \gamma^{5} \Psi=-\frac{1}{\sqrt{\pi}} \partial^{\mu} \Phi,
$$

where $\Sigma, \tilde{\eta}$ and $\Phi$ are so far unspecified scalar fields. In the $\partial_{\mu} A^{\mu}=0$ gauge, one finds $\partial_{\mu} \partial^{\mu} \tilde{\eta}=0$ and $F_{\mu \nu}=\frac{\sqrt{\pi}}{e} \epsilon_{\mu \nu} \partial_{\rho} \partial^{\rho} \Sigma$. From the anomalous divergence of the axial current

$$
\partial_{\mu} J_{5}^{\mu}=\frac{e}{2 \pi} e_{\mu \nu} F^{\mu \nu}
$$

one concludes that $\partial_{\mu} \partial^{\mu} \Phi=\partial_{\mu} \partial^{\mu} \Sigma$ or $\Phi=\Sigma+h$ with the free massless field $h$ obeying $\partial_{\mu} \partial^{\mu} h=0$. Then the vector current is

$$
J_{\mu}=-\frac{1}{\sqrt{\pi}} \tilde{\partial}_{\mu}+L_{\mu}, \quad L_{\mu}=-\frac{1}{\sqrt{\pi}} \tilde{\partial}_{\mu} h .
$$

From the Maxwell eqs.

$$
\tilde{\partial}^{\nu}\left(\partial_{\rho} \partial^{\rho}+\frac{e^{2}}{\pi} \Sigma\right)-\frac{e^{2}}{\sqrt{\pi}} L^{\nu}=0
$$

one concludes that $\tilde{\partial}^{\mu} L_{\mu}=0$ or

$$
\left(\partial_{\rho} \partial^{\rho}+\frac{e^{2}}{\pi}\right) \Sigma=0
$$

One component of the gauge field, namely $\Sigma(x)$, became massive. For consistency reasons, $L_{\mu}$ can vanish only weakly, $\left\langle\psi\left|L_{\mu}(x)\right| \psi\right\rangle=0$. With the above Ansatz for $A^{\mu}$, Dirac eq. becomes $i \gamma^{\mu} \partial_{\mu} \Psi=$ $-\sqrt{\pi} \gamma^{\mu} \gamma^{5} \partial_{\mu}(\Sigma+\eta) \Psi$ with the solution

$$
\Psi(x)=: e^{i \sqrt{\pi} \gamma^{5}(\Sigma(x)+\eta(x))}: \psi(x) .
$$

Calculation of the currents via the point-splitting yields identification $h(x)=\eta(x)+\varphi(x)$, where $\varphi(x)$ is the "potential" (integrated current) of the free currents. From $\left[A^{1}\left(x^{1}\right), \pi_{1}\left(y^{1}\right)\right]=i \delta\left(x^{1}-y^{1}\right)$ and with $\pi_{1}=F^{01}=\frac{e}{\sqrt{\pi}} \Sigma$ one gets the equal-time commutator $\left[\Sigma\left(x^{1}\right), \partial_{0} \Sigma\left(y^{1}\right)\right]=i \delta\left(x^{1}-y^{1}\right)$ of a canonical scalar field. Inserting Eq.(26) to the original Lagrangian, we derive the physical part of the Hamiltonian as :

$$
H=\int_{-\infty}^{+\infty} \mathrm{d} x^{1}\left[-i \psi^{\dagger} \alpha^{1} \partial_{1} \psi+\frac{1}{2} \frac{e^{2}}{\pi} \Sigma^{2}\right] .
$$

This Hamiltonian is non-diagonal when expressed in terms of Fock operators of $\Sigma(x)$. A Bogoliubov transformation is necessary. A coherent-type of vacuum state will be obtained as the true vacuum.

An interesting aspect of the model is its vacuum degeneracy and the theta vacuum. In the work 2 the mechanism generating multiple vacua is based on "spurion" operators. These however have 
been shown to be an artifact of the incorrect treatment of residual gauge freedom [3. What can be the true mechanism of the vacuum degeneracy in the Scwinger model? We believe that it is the presence of a gauge zero mode in the finite-volume treatment of the model 14 together with a quantum implementation of residual invariance under large gauge transformations as desribed in 15 . This leads us naturally to the finite-volume reformulation of our approach outlined in the first part of this section.

\section{Spontaneous symmetry breaking in LF theory with fermions}

At the LC workshop in Valencia, Marvin Weinstein criticised the way how the LF theory describes spontaneous symmetry breaking, saying that Goldstone (or hidden) symmetry is a chalenge for LF theory. Where is vacuum degeneracy? Actually the latter can be described if one takes into account a mechanism based on the presence of dynamical fermion zero modes [16. $O(2)$-symmteric sigma model provides us with a good example. Its Hamiltonian is symmetric under axial-vector transformations

$$
\Psi_{+}(x) \rightarrow e^{-i \beta \gamma^{5}} \Psi_{+}(x)=V(\beta) \Psi_{+}(x) V^{-1}(\beta), \quad V(\beta)=\exp \left\{-i \beta Q_{5}\right\}, \quad Q_{5}=\int_{V} d^{3} \underline{x} J_{5}^{+}(x) .
$$

The operator of the axial charge will not annihilate the LF vacuum since in addition to the normalmode part (which annihilates it) it contains also the zero-mode term, $Q^{5}=Q_{N}^{5}+Q_{0}^{5}$, where

$$
Q_{0}^{5}=\sum_{p_{\perp}, s} 2 s\left[\left(b_{0}^{\dagger}\left(p_{\perp}, s\right) d_{0}^{\dagger}\left(-p_{\perp},-s\right)+H . c .\right)+b_{0}^{\dagger}\left(p_{\perp}, s\right) b_{0}\left(p_{\perp}, s\right)-d_{0}^{\dagger}\left(p_{\perp}, s\right) d_{0}\left(p_{\perp}, s\right)\right] .
$$

The term $b_{0}^{\dagger}\left(p_{\perp}\right) d_{0}^{\dagger}\left(-p_{\perp}\right)$ will generate an infinite set of degenerate vacuum states. One has all properties for deriving the Goldstone theorem in the usual way.

We conclude with the statement that the realm of exactly solvable models still offers us certain surprises and room for improvement. And that degenerate vacua exist in the LF formalism in spite of its kinematically defined vacuum state.

Acknowledgements This work has been supported by the grant VEGA No. 2/0070/2009 and by the Slovak CERN Commission. The author also thanks Pierre Grangé for fruitfull discussions.

\section{References}

1. Klaiber B. (1968) The Thirring model, In: Lectures in Theoretical Physics, Vol. Xa, Boulder 1976: 141-176

2. Lowenstein J. H. and J. A. Swieca J. A. (1971) Quantum electrodynamics in two-dimensions, Ann. Phys. 68: $172-195$

3. Morchio G. and Strocchi F. (1988) The Schwinger Model Revisited, Ann. Phys. 188: 217-238

4. L. Martinovic and P. Grange, work in preparation

5. Schroer B. (1963) Infrateilchen in der Quantenfeldtheorie, Fort. der Physik 11: 1-31

6. Thirring W. (1958) A soluble relativistic field theory, Ann. Phys. 3: 91-112

7. Federbush K. (1961) A Two-Dimensional Relativistic Field Theory, Phys. Rev. 121: 1247-1249

8. Wightman A. S. (1964) Introduction to Some Aspects of the Relativistic Dynamics of Quantized fields, In: Cargese Lectures in Theoretical Physics, Gordon and Breach, new York 1967: 171

9. Schroer B. and Truong T. (1977) Equivalence of Sine-Gordon and The Thirring Model and Cummulative Mass effects, Phys. Rev. D15: 1684-1693

10. Gross D. and Neveu A. (1974) Dynamical symmetry breaking in asymptotically free field theories, Phys. Rev. D 10: 3235-3253

11. Thirring W. and Wess J. (1964) Solution of a field theory model in one space and one time dimensions, Ann. Phys. 27: 331-337

12. Haller K. and Lim-Lombridas E. (1994) Quantum gauge equivalence in QED, Found. of Phys. 24: 217-247

13. Abdalla E., Abdalla M.C.B and Rothe K. D. (2001) Nonperturbative methods in two-dimensional quantum field theory, World Scientific, Singapore, $832 \mathrm{p}$.

14. Hetrick J. E. and Hosotani Y. (1988) QED on a circle, Phys. Rev. D 38: 2621-2624

15. Martinovic L. (2001) Gauge symmetry and the light-front vacuum structure, Phys. Lett. B509: 355-364

16. Martinovic L. and Vary J. P. (2001), Fermionic zero modes and spontaneous symmtery breaking on the light front, Phys. Rev. D64: 15016-15020 